\title{
Copula techniques in wireless communications
}

\author{
J. Kitchen ${ }^{1} \quad$ W. Moran $^{2}$
}

(Received 20 January 2010; revised 19 July 2010)

\begin{abstract}
Copula techniques were originally developed as a method for modelling data dependence in financial applications and are proving useful in many other fields. We show how the Copula concept may be exploited to model dependence in wireless communications problems. In particular we consider multipath correlation, with signal fading, in a wireless propagation medium. The Copula approach is also considered for the purpose of separating signals that have become dependent in such propagation scenarios and we investigate methods for Blind Source Separation that provide alternatives to the popular Independent Component Analysis approach. Our approach to the Blind Source Separation problem forms an objective function based on the copula parameter of the dependence structure, then a transformation is sought which inverts the function producing the dependence and which yields an independent copula. This approach has the potential to provide a robust and easily applied technique for isolating wireless communications signals in a wide range of propagation scenarios.
\end{abstract}

http://anziamj.austms.org.au/ojs/index.php/ANZIAMJ/article/view/2451 gives this article, (c) Austral. Mathematical Soc. 2010. Published August 2, 2010. ISSN 1446-8735. (Print two pages per sheet of paper.) 


\section{Contents}

1 Introduction

C527

2 Copula theory

C528

3 Correlated fading

C528

4 Blind source separation

C534

5 Summary

C538

References

C538

\section{Introduction}

The theory of copulas was originally developed as a means of incorporating dependence between random variables in the field of finance and there are many useful introductory texts on the subject of copulas, for example see the text by Nelsen [9]. A topical and highly active area in the field of communications theory is that of Multi-Input Multi-Output (MIMO) wireless communications systems, where communication takes place between a transmitter array and a receiver array. Two wireless communications challenges are considered here as potential candidates for the application of copula techniques: modelling signal correlation and propagation effects, Blind Source Separation (BSS).

The Rayleigh distribution has been a long term standard; however, thanks to its wide versatility and analytic tractability, the Nakagami-m distribution has become popular for modelling wireless fading scenarios $[1,2]$. Techniques for modelling data correlation in the MIMO case tended to be quite involved and hence difficult to implement and computationally demanding. A simple and intuitive approach is desirable. 
There are several algorithms developed for the purpose of separating mixtures of independent sources. For many of these algorithms to be successful, the original independent sources must have non-Gaussian marginal probability densities. Copula methods offer an alternative approach that does not depend on the source distributions but which instead exploits the structure of the dependence between the sources.

\section{Copula theory}

A copula can be briefly described as a function that connects one dimensional marginal distributions through a multivariate distribution and may therefore be seen as a means for deriving multivariate distributions with any desired dependence incorporated. There are several well-known copula families which are described in the literature $[9$, e.g.]. The basis for the theory of copulas stems from Sklar's Theorem [9] which states: An m-dimensional copula is a function $C$ from the unit $m$-cube $[0,1]^{m}$ to the unit interval $[0,1]$ and satisfies certain conditions. For an $\mathrm{m}$-variate function $\mathrm{F}$, the copula associated with $\mathrm{F}$ is a distribution function $C:[0,1]^{\mathrm{m}} \rightarrow[0,1]$ that satisfies

$$
F\left(y_{1}, \ldots, y_{m}\right)=C\left(F_{1}\left(y_{1}\right), \ldots, F_{m}\left(y_{m}\right) ; \theta\right),
$$

where $\theta$ is a parameter of the copula called the dependence parameter, which measures dependence between the marginals. We make use of two copulae in this study: multivariate Gaussian for modelling dependence, and the independent (or product copula) for BSS purposes.

\section{$3 \quad$ Correlated fading}

We develop the MIMO wireless propagation model and show how a copula may be employed to account for dependence or correlation in the propagation channel. In wireless communications, fading is the attenuation that a 


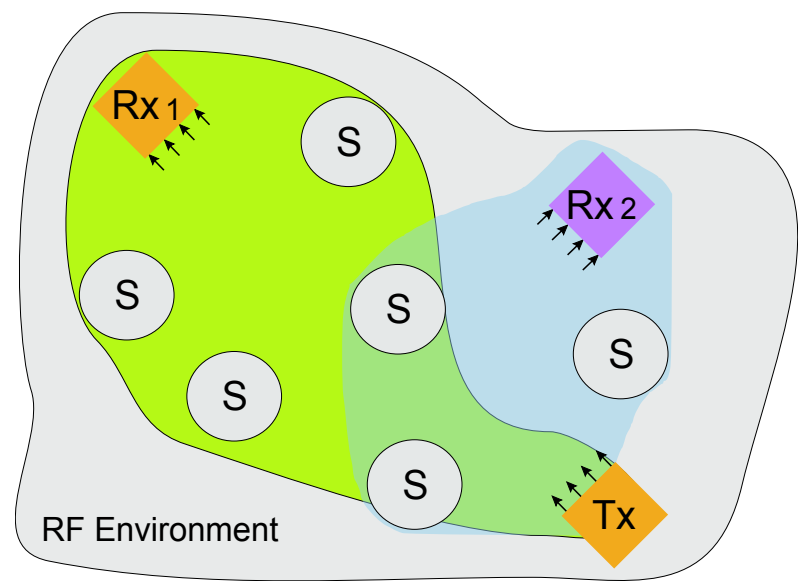

Figure 1: MIMO RF Scenario. Propagation between TX and RX1 is indicated in green. Propagation between TX and RX2 is indicated in light blue. RF scattering is represented by scatterers $\mathrm{S}$.

signal experiences when passing through a propagation medium and is often modelled as a random process. Reflectors in the environment surrounding a transmitter and receiver create multiple paths that a transmitted signal traverses. As a result, a receiver sees the superposition of multiple copies of the transmitted signal. Each signal copy will experience differences in attenuation, delay and phase shift. A number of different probability distributions have been employed by researchers to simulate multipath fading effects including: Rayleigh, Rician, Nakagami-m.

Figure 1 illustrates a MIMO wireless Radio Frequency (RF) scenario with one transmitter array (TX) and two independent receiver arrays (RX1 and Rx2). The whole RF environment is represented in grey. RF propagation between TX and RX1 due to line-of-sight signals and RF scatterers in the environment is shown in green. RF propagation between $\mathrm{TX}$ and $\mathrm{RX} 2$ is shown in light blue. This picture could represent a communications broadcast scenario, where both of the receivers are intended to receive the signals from the transmitter, or a surveillance scenario, where one of the receivers is not the intended recipient 
of the signals. A simple linear mixing model that is commonly used for MIMO simulations is $\mathbf{Y}=\mathbf{A X}+\mathbf{W}$, where $\mathbf{Y}$ is the $\mathbf{m} \times \mathfrak{n}$ received signal matrix, $\mathbf{X}$ is the transmitted source matrix, $\mathbf{W}$ is an additive receiver noise matrix and $\mathbf{A}$ is an $\mathbf{m} \times \mathfrak{m}$ channel gain or mixing matrix between the transmitter and receiver. The array dimension is $m$ and $n$ is the number of samples in a given block of time. We make the following assumptions: $\mathbf{X}$ is an iid $\mathrm{m} \times \mathrm{n}$ random source matrix with zero mean and $\operatorname{var}\left\{x_{i, j}\right\}=\sigma_{x}^{2} ; \mathbf{W}$ is an iid $\mathfrak{m} \times \mathfrak{n}$ random Gaussian noise matrix with zero mean; and $\operatorname{var}\left\{\boldsymbol{w}_{i, j}\right\}=\sigma_{w}^{2}$. Dependence may be introduced at the transmitter array, the receiver array, within the propagation channel, or any combination of these. We require a flexible technique that will allow us to change the fading distribution of the channel coefficients and incorporate dependence between them. This may be readily achieved through the use of copulae. To obtain a sequence of random fading channel coefficients that are dependent we take the following approach.

- Let $\mathbf{a}=\operatorname{vec}(\mathbf{A})$; that is, vector $\mathbf{a}$ is an $m^{2} \times 1$ vector with elements $a_{1}, a_{2}, \ldots, a_{m^{2}}$ that are independent random variables distributed according to whatever fading we require. This could be a mix of Rayleigh, Rician or Nakagami-m random variables.

- Let $a_{i} \sim P_{i}\left(a_{i}\right)$; that is, $a_{i}$ is distributed with distribution function $P_{i}\left(a_{i}\right)$ and let the desired joint distribution for a be $P(\mathbf{a})=P\left(a_{1}, a_{2}, \ldots, a_{m^{2}}\right)$. The copula is defined for $P(\mathbf{a})$ as $C(\mathbf{u})=P(\mathbf{a})$, where $u_{i}=P_{i}\left(a_{i}\right)$ or, alternatively, $a_{i}=P_{i}^{-1}\left(u_{i}\right)$ and the $u_{i}$ are uniformly distributed variates.

- The inverse functions of the marginal distributions are $P_{1}^{-1}, \ldots, P_{m^{2}}^{-1}$ so that $a_{1}=P_{1}^{-1}\left(u_{1}\right), a_{2}=P_{2}^{-1}\left(u_{2}\right), \ldots, a_{m^{2}}=P_{m^{2}}^{-1}\left(u_{m^{2}}\right)$, where $\mathfrak{u}_{1}, \ldots, \mathfrak{u}_{\mathrm{m}^{2}}$ are uniformly distributed variates. Hence,

$$
\mathrm{P}(\mathbf{a})=\mathrm{P}\left[\mathrm{P}_{1}^{-1}\left(\mathrm{u}_{1}\right), \ldots, \mathrm{P}_{\mathrm{m}^{2}}^{-1}\left(\mathrm{u}_{\mathrm{m}^{2}}\right)\right]=\mathrm{C}\left(\mathrm{u}_{1}, \ldots, \mathrm{u}_{\mathrm{m}^{2}}\right)=\mathrm{C}(\mathbf{u}),
$$

where $\mathbf{C}(\mathbf{u})$ is the copula that must be chosen to link the marginals.

In short, the procedure for generating a channel matrix sequence, with dependence, is the following. 
1. Choose an appropriate multivariate copula and generate a matrix, which is $m^{2} \times n$, of dependent uniformly distributed random variables, where each row corresponds to one of the channel matrix coefficients.

2. Choose a fading distribution for each of the elements (rows) of the matrix and apply the inverse function so that a matrix of dependent random variables with the desired distributions is obtained.

3. Convert the matrix to a sequence of $m \times m$ matrices using the inverse of the vec $(\cdot)$ operation for each column of the $m^{2} \times n$ matrix.

To obtain a complex mixing matrix we assume that the real components are independent of the imaginary components and repeat the above procedure to obtain two real matrices. This produces a random matrix for the fading amplitude; however, we need to consider the distribution of the phase of the coefficients. Let $\mathbf{A}_{R}$ and $\mathbf{A}_{I}$ represent, respectively, the real and imaginary parts of $\mathbf{A}$, obtained from two repetitions of the above procedure, then we obtain the correct phase distribution in forming the complex mixing matrix

$$
\mathbf{A}=\mathbf{A}_{\mathrm{R}} \odot \operatorname{sign}\left(\mathbf{B}_{\mathrm{R}}\right)+\mathbf{j} \mathbf{A}_{\mathrm{I}} \odot \operatorname{sign}\left(\mathbf{B}_{\mathrm{I}}\right),
$$

where $\mathbf{B}_{R}$ and $\mathbf{B}_{I}$ are two matrices with iid normally distributed components and which are the same size as $\mathbf{A}_{R}$ and $\mathbf{A}_{I}$ respectively. Complex values are formed using the imaginary unit $j=\sqrt{-1}$ and $\odot$ is the Hadamard or elementwise product. The true phase and amplitude expressions for the Nakagami-m distribution are provided by Yacoub, Fraidenraich and Santos Filho [11] and are used in the simulations for comparison.

An Octave $^{1}$ (a free alternative to Matlab) implementation for dependent fading channel generation was developed. The code allows for marginal channel distributions to be chosen from either the Rayleigh or Nakagami-m distributions. The multivariate channel copula may be selected from the: Normal, Student-t, Clayton, Frank or Gumbel distributions. The Nakagami$m$ distribution can be obtained in two different ways: inverse distribution

\footnotetext{
${ }^{1}$ http://www.gnu.org/software/octave/
} 

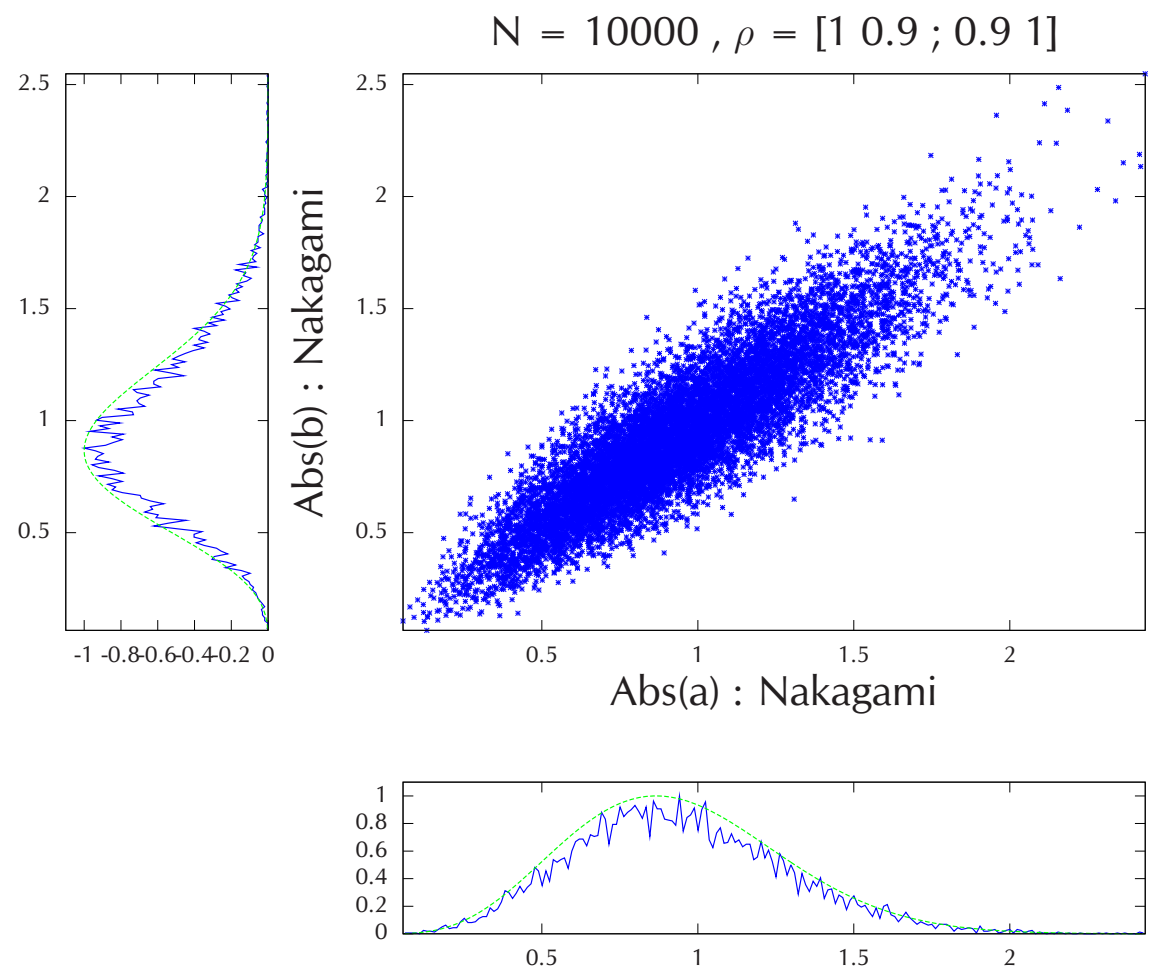

Figure 2: Amplitude distributions, Nakagami fading, Gaussian copula.

approximation [2] or inverse gamma distribution [12] then take the square root of the result.

Simulations have been performed to demonstrate this method and results are shown in Figures 2 and 3. In the simulations two elements of a channel matrix are studied. A sequence of 10000 instances of the pair of elements is generated where the elements follow a Nakagami-m distribution and a Gaussian copula is utilised, with a correlation matrix where the cross-correlation terms $=0.9$ (that is, highly dependent). Figure 2 shows a scatter plot of the correlated amplitudes of the two elements. The associated histogram plots compare the 

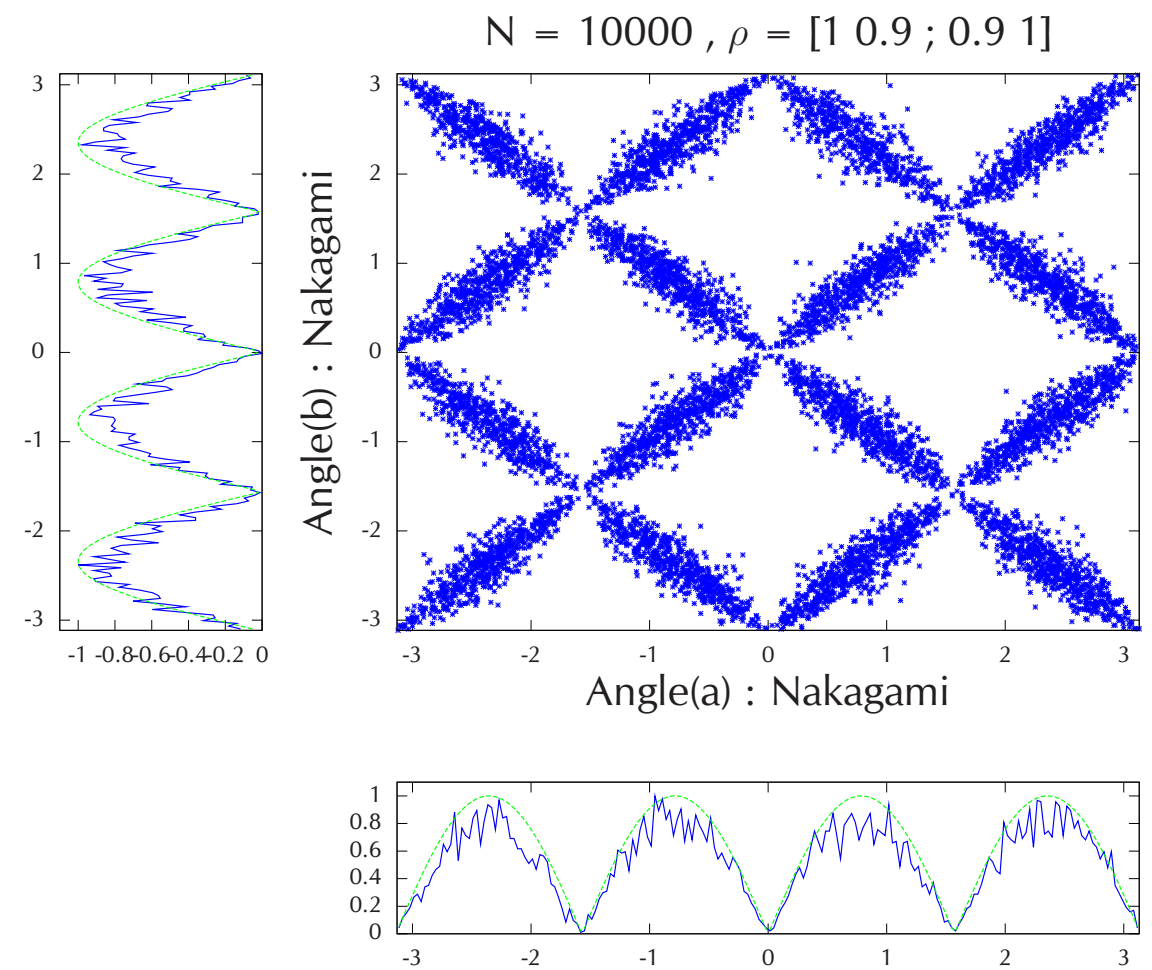

Figure 3: Phase distributions, Nakagami fading, Gaussian copula.

amplitude distributions with the theoretical Nakagami amplitude distribution. Figure 3 shows the correlated phases of the two elements. The associated histogram plots compare the phase distributions with the theoretical Nakagami phase distribution. 


\section{Blind source separation}

We now consider the problem of receiving MIMO signals that have passed through an unknown channel. The challenge is to estimate both the channel mixing matrix and the source signals. Problems of this type are known as blind source separation problems and many techniques have been developed that attempt to separate a multivariate signal into subcomponents that are mutually independent. Many of these techniques rely on objective function tests for non-Gaussianity in the estimated components. The independent components are identifiable up to a permutation and scaling of the sources. A popular Independent Component Analysis (ICA) implementation is the FastICA algorithm [6]. Copula based approaches have been previously proposed $[3,7]$ which have preprocessing steps in common with ICA algorithms. In the case of a multivariate Gaussian copula the copula parameter is the correlation matrix so that the sources will have been resolved when there is zero correlation between separated components. Alternative tests for independence include Kendall's $\tau$ [4] that test for dependence between the rankings of the components. The RADICAL algorithm [8] uses an entropy-based estimator and has also been implemented in our simulations. The algorithms proceed with the following steps.

- Center the observed data - subtract the mean and normalise (unit power) the observed mixture power.

- Whiten observations - via Eigenvalue Decomposition (EVD). This procedure converts the observation covariance matrix to an identity matrix and reduces the search space to a search for a unitary transformation.

- Find a unitary (complex data) or orthogonal (real data) transformation that minimises an objective function: kurtosis, negentropy, copula parameter.

We simulated the MIMO scenario where there is a transmitter array with two elements and a receiver array with two elements so that the channel is 
represented by a $2 \times 2$ matrix. Random message blocks of size $2 \times 500$ were generated with the assumption that the channel matrix remained constant for this block length. The distribution of the independent sources was controlled by employing the Generalised Gaussian distribution [10] parametrised by $\alpha$, where the distribution is Gaussian when $\alpha=2$. When $\alpha<2$ the distribution has a positive kurtosis and when $\alpha>2$ the distribution has a negative kurtosis. For each value of $\alpha, 100$ instances were generated of the $2 \times 500$ message block and the $2 \times 2$ channel matrix. A Gaussian noise matrix was added so that the input signal-to-noise ratio (snr) was $10 \mathrm{~dB}$. The average performance of the separation algorithms was then calculated as the mean output snr over the 100 repetitions. Ambiguities in scale and permutation were taken into account in the simulations. In the real data case, after prewhitening, the algorithms must find an orthogonal $2 \times 2$ matrix that maximises the estimated source independence. This is equivalent to finding the angle for a $2 \mathrm{D}$ rotation matrix. However, in the $2 \mathrm{D}$ complex data case, the unitary matrix is formed from a rotation angle and three phases and so there are four degrees of freedom that must be optimised [5].

Figure 4 compares the simulation results for real data. The MLE assumes that the mixing matrix is known a priori. The FasticA results have a minimum when the Generalised Gaussian distribution parameter $\alpha=2$, confirming the well-known fact that this algorithm has difficulty in separating a mixture of Gaussian sources. However, as $|\alpha-2|$ increases, FastICA is better able to separate the sources. Results from the RADICAL algorithm are similar to those from FasticA for $\alpha<2$ but degrade when $\alpha>2$. Results from the Kendall's $\tau$ and correlation algorithms are poor but are clearly independent of the source distribution. Figure 5 compares the results for complex data. As for the real data case, the FastICA results have a minimum when $\alpha=2$. Results from the Kendall's $\tau$ and correlation algorithms are poor but again are independent of the source distribution. When the sources are close to Gaussian, the Kendall's $\tau$ and correlation algorithms appear to perform marginally better than FasticA. 
(RI) Output SNR Vs Non-Gaussianity, snr $=10 \mathrm{~dB}, \mathrm{~N}=500$, Array $=2$.

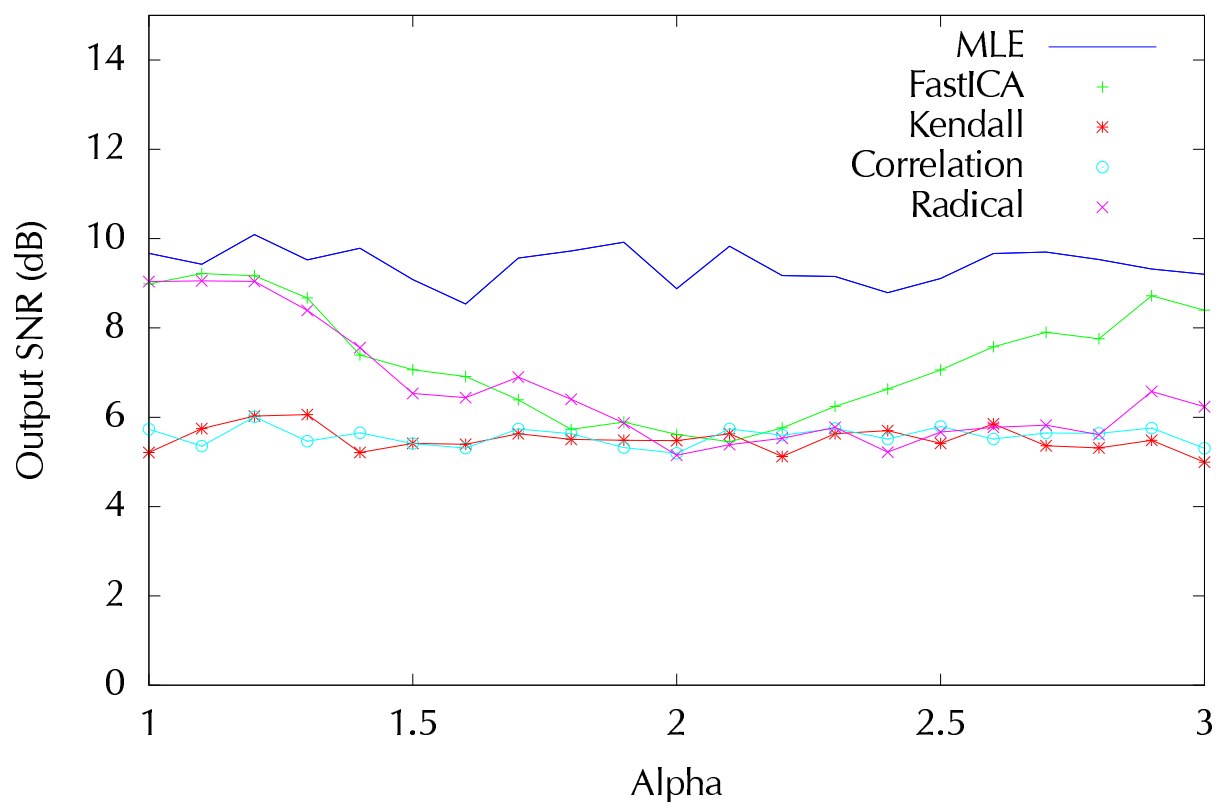

FiguRE 4: Source separation performance, real data. 
(Cx) Output SNR Vs Non-Gaussianity, snr $=10 \mathrm{~dB}, \mathrm{~N}=500$, Array $=2$.

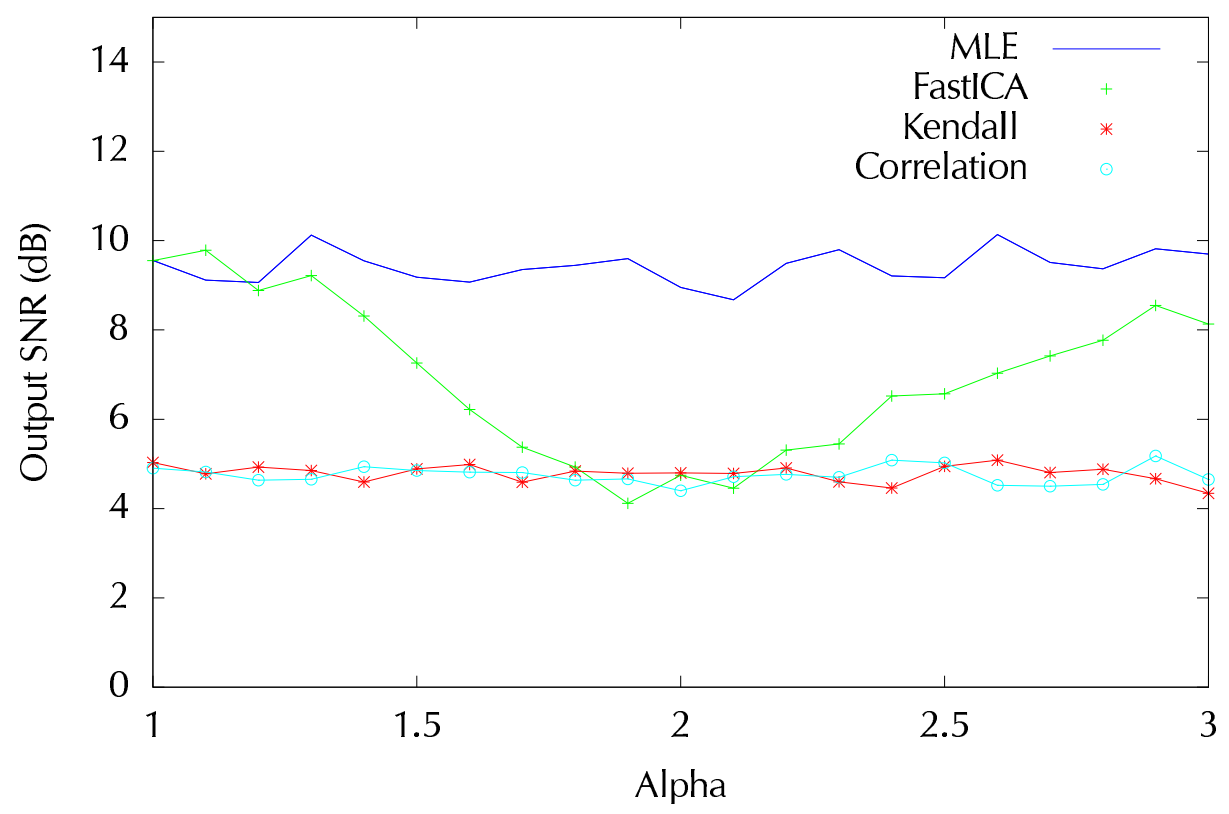

Figure 5: Source separation performance, complex data. 


\section{Summary}

Copula theory has been shown to provide a practical and intuitive approach for modelling dependence in MIMO wireless channel simulations and, with further work, may be adapted to overcome some limitations inherent in ICA, if a reliable test for independence can be found.

\section{References}

[1] M.-S. Alouini, A. Abdi, and M. Kaveh. Sum of gamma variates and performance of wireless communication systems over Nakagami-fading channels. Vehicular Technology, IEEE Transactions on, 50(6):1471-1480, November 2001. doi:10.1109/25.966578 C527

[2] N. C. Beaulieu and C. Cheng. an efficient procedure for Nakagami-m fading simulation. In Global Telecommunications Conference, 2001. GLOBECOM '01. IEEE, volume 6, pages 3336-3342, November 2001. doi:10.1109/GLOCOM.2001.966304 C527, C532

[3] Ray-Bing Chen, Meihui Guo, Wolfgang Härdle, and Shih-Feng Huang. Independent component analysis via copula techniques. SFB 649 Discussion Papers SFB649DP2008-004, Sonderforschungsbereich 649, Humboldt University, Berlin, Germany, January 2008. http://ideas.repec.org/p/hum/wpaper/sfb649dp2008-004.html C534

[4] David Christensen. Fast algorithms for the calculation of Kendall's $\tau$. Computational Statistics, 20(1):51-62, March 2005. doi:10.1007/BF02736122 C534

[5] P. Diţă. Factorization of unitary matrices. Journal of Physics A: Mathematical and General, 36(11):2781-2789, March 2003. doi:10.1088/0305-4470/36/11/309 C535 
[6] A. Hyvärinen. Fast and robust fixed-point algorithms for independent component analysis. Neural Networks, IEEE Transactions on, 10(3):626-634, May 1999. doi:10.1109/72.761722 C534

[7] Jian Ma and Zengqi Sun. Copula component analysis. CoRR, 2007. http://arxiv.org/abs/cs/0703095 C534

[8] Erik G. Miller and John W. Fisher III. ICA using spacings estimates of entropy. Journal of Machine Learning Research, 4:1271-1295, December 2003.

http://jmlr.csail.mit.edu/papers/v4/learned-miller03a.html C534

[9] R. B. Nelsen. An introduction to copulas. Springer-Verlag New York, Inc., 1999. doi:10.1007/0-387-28678-0 C527, C528

[10] P. Tichavský, Z. Koldovský, and E. Oja. Performance analysis of the FastICA algorithm and Cramér-Rao bounds for linear independent component analysis. IEEE Trans. on Signal Processing, April 2006. doi:10.1109/TSP.2006.870561 C535

[11] M. D. Yacoub, G. Fraidenraich, and J. C. S. Santos Filho. Nakagami-m phase-envelope joint distribution. Electronics Letters, 41(5):259-261, March 2005. doi:10.1049/el:20057014 C531

[12] Q. T. Zhang. A decomposition technique for efficient generation of correlated nakagami fading channels. Selected Areas in Communications, IEEE Journal on, 18(11):2385-2392, November 2000. doi:10.1109/49.895043 C532

\section{Author addresses}

1. J. Kitchen, Defence Science and Technology Organisation, South Australia, Australia. mailto: john.kitchen@dsto.defence.gov.au 
2. W. Moran, Department of Electrical and Electronic Engineering, University of Melbourne, Victoria, Australia. 